\title{
Oral curcumin in elective abdominal aortic aneurysm repair: a multicentre randomized controlled trial
}

Amit X. Garg MD, P.J. Devereaux MD, Andrew Hill MD, Manish Sood MD, Bharat Aggarwal PhD, Luc Dubois MD, Swapnil Hiremath MD, Randolph Guzman MD, Vikram Iyer MD, Matthew James MD, Eric McArthur MSc, Louise Moist MD, Georges Ouellet MD, Chirag R. Parikh MD, Virginia Schumann, Sumit Sharan MD, Heather Thiessen-Philbrook MMath, Sheldon Tobe MD, Ron Wald MD, Michael Walsh MD, Matthew Weir MD, Neesh Pannu MD; Curcumin AAA AKI Investigators

— Cite as: CMAJ 2018 October 29;190:E1273-80. doi: 10.1503/cmaj.180510

CMAJ Podcasts: author interview at https://soundcloud.com/cmajpodcasts/180510-res

See related article at www.cmaj.ca/lookup/doi/10.1503/cmaj.181358

\begin{abstract}
BACKGROUND: Curcumin, a popular herbal supplement from the plant turmeric, has prevented ischemic reperfusion and toxin-induced injury in many animal studies and a singlecentre randomized human trial. We sought to test whether perioperative oral curcumin (compared with placebo) affects the inflammatory response and risk of postrepair complications after elective abdominal aortic aneurysm repair in humans.
\end{abstract}

METHODS: We conducted a parallelgroup, randomized, placebo-controlled trial of patients from 10 hospitals in Canada who were scheduled to undergo elective repair of an unruptured abdominal aortic aneurysm (November 2011 to November 2014). Patients in the treat- ment group received perioperative oral curcumin (2000-mg doses 8 times over $4 \mathrm{~d})$. Patients, health care providers and local research staff were unaware of the treatment assignment. The primary outcomes were median concentrations of 4 biomarkers indicating injury and inflammation (postoperative urine interleukin-18 and perioperative rise in serum creatinine, plasma $\mathrm{N}$-terminal pro-B-type natriuretic peptide and plasma highsensitivity C-reactive protein).

RESULTS: Baseline characteristics were similar in the 2 groups (606 patients overall; median age $76 \mathrm{yr}$ ). More than $85 \%$ of patients in each group took more than $80 \%$ of their scheduled capsules. Neither curcumin nor placebo significantly affected any of the 4 biomarkers ( $p>0.05$ for all comparisons). Regarding the secondary outcomes, there was a higher risk of acute kidney injury with curcumin than with placebo $(17 \%$ v. $10 \%, p=$ 0.01 ), but no between-group difference in the median length of hospital stay ( 5 v. 5 days, $p>0.9$ ) or the risk of clinical events ( $9 \%$ v. $9 \%, p=0.9$ ).

INTERPRETATION: Curcumin had no beneficial effects when used in elective abdominal aortic aneurysm repair. These findings emphasize the importance of testing turmeric and curcumin before espousing their health benefits, as is currently done in the popular media. Trial registration: ClinicalTrials.gov, no. NCT01225094.
M any modern medicines come from plants. ${ }^{1,2}$ Turmeric, a plant in the ginger family that is native to South Asia, has been used for centuries as a spice and traditional medicine. The active medicinal ingredient, curcumin, is a polyphenol that constitutes about 3\% of turmeric by weight. Sales of oral curcumin as a natural health product continue to rise worldwide, ${ }^{3}$ supported by many biological studies that show its antioxidant and anti-inflammatory properties. ${ }^{4-6}$ For example, more than 35 animal studies have shown that curcumin reduces the risk of ischemic reperfusion and toxin-induced injury to the kidney (see section 1 of Appendix 1, available at www.cmaj.ca/ lookup/suppl/doi:10.1503/cmaj.180510/-/DC1, which includes animal models of abdominal aorta cross-clamping and contrastinduced injury). ${ }^{7,8}$ Curcumin has the potential to prevent organ injury from surgery by preserving endothelial function, reducing inflammation and limiting oxidative stress (see section 2 of 
Appendix 1$).{ }^{9}$ In a randomized controlled trial of 121 consecutive patients undergoing coronary artery bypass graft surgery at a single centre in Thailand, oral curcumin, relative to placebo, reduced the risk of postoperative myocardial infarction and lowered concentrations of plasma biomarkers for inflammation, oxidation and injury. Specifically, it attenuated the perioperative rise in the plasma concentration of $\mathrm{N}$-terminal pro-B-type natriuretic peptide (NT-pro-BNP), C-reactive protein and malondialdehyde). ${ }^{10}$

Elective abdominal aortic aneurysm repair, a common operation worldwide, can injure the kidneys and other organs through ischemia from aortic cross-clamping (open procedure) or an intravascular contrast agent (endovascular repair). We conducted this multicentre randomized, placebo-controlled trial to test whether perioperative oral curcumin alters a biomarker panel assessing injury and the inflammatory response or the risk of postrepair complications after elective abdominal aortic aneurysm repair.

\section{Methods}

We conducted a randomized, parallel-group, placebocontrolled trial with blinding of patients, providers and assessors. Participants were recruited at 10 academic hospitals in 4 provinces (listed in section 3 of Appendix 1).

\section{Participants}

The complete eligibility criteria are reported in section 3 of Appendix 1. In brief, eligible patents were scheduled for elective repair of an abdominal aortic aneurysm, planned as either an open or an endovascular procedure. Patients scheduled for an endovascular procedure had to have at least 1 preoperative risk factor for surgical complications (diabetes mellitus, older than 70 years of age or elevated serum creatinine concentration, defined by value $>177 \mu \mathrm{mol} / \mathrm{L}$ in men or $>146 \mu \mathrm{mol} / \mathrm{L}$ in women). All participants provided written informed consent before randomization.

\section{Randomization}

Research personnel at the participating sites entered demographic and baseline clinical characteristics into a secure webbased database system that also provided randomization. Baseline data were checked to confirm each patient's eligibility, and the system then assigned the participant to curcumin or placebo therapy, with assignments in a 1:1 ratio stratified by centre. The sequence of assignment was determined by the webbased, computer-generated blocked random number table.

\section{Blinding}

Participants, their health care providers and the data collectors were unaware of the group assignment. The placebo capsules looked, smelled and tasted the same as the curcumin capsules, and were made of yellow food colouring, gelatin and cellulose.

\section{Interventions}

Patients were assigned to take curcumin or placebo according to the trial regimen, which is described and justified in section 4 of Appendix 1. All specifications for the curcumin and placebo capsules, including tests confirming stability of the capsules, were submitted to Health Canada. The curcumin capsules contained 95\% curcumin extracted from the rhizome of Curcuma longa (turmeric) and were free of impurities. Participants randomly assigned to the intervention group were scheduled to receive a total of $4000 \mathrm{mg}$ of curcumin per day in 2 divided doses for 2 days before the repair, followed by $2000 \mathrm{mg}$ the morning of the repair, $2000 \mathrm{mg}$ on call to the operating room, $2000 \mathrm{mg} 6$ hours after surgery and $2000 \mathrm{mg}$ the next morning. We did not impose any dietary restrictions, as foods spiced with turmeric provide exceedingly small amounts of curcumin. ${ }^{11,12}$ We did not monitor participants' use of over-the-counter curcumin or turmeric supplements, but we did ask all participants to refrain from taking such supplements.

\section{Outcome measures}

Full definitions of the trial outcomes are provided in section 5 of Appendix 1. Preoperative blood samples were collected a median of 9 (interquartile range [IQR] 5 to 17) days before the repair. Postoperative urine and blood samples for biomarkers other than serum creatinine were collected a median of 1 (IQR 1 to 1 ) hour after the end of the repair and were used for postoperative biomarker measurement. Serum creatinine concentrations were measured for the first 2 days after surgery; any postoperative value greater than $9 \mu \mathrm{mol} / \mathrm{L}(0.1 \mathrm{mg} / \mathrm{dL})$ above the preoperative value resulted in at least 2 more days of measurements, even if the patient was discharged from hospital. The postoperative serum creatinine used in the primary outcome was the highest value in the 7 days after surgery. Patients were contacted 30 days after surgery to determine whether clinical events had occurred after hospital discharge, and again 90 days after surgery to obtain a serum creatinine value and to record vital status.

The primary outcomes were 4 biomarkers to assess different aspects of injury and the inflammatory response in the kidney and other organs: postoperative concentration of urine interleukin-18 and perioperative (postoperative minus preoperative) rise in the concentration of serum creatinine, plasma NT-pro-BNP and plasma high-sensitivity C-reactive protein.

Interleukin-18, which mediates kidney ischemic reperfusion injury in animals, is synthesized by several tissues, including proximal kidney tubular epithelial cells. ${ }^{13,14}$ The preoperative concentration of urine interleukin-18 is constitutively low and has no prognostic significance. A high concentration of urine interleukin-18 in the hours after cardiac surgery is associated with higher risks of clinical acute kidney injury and death and also with a longer hospital length of stay..$^{15}$ Rising serum creatinine concentrations in the days after an insult are used in clinical practice to diagnose acute kidney injury. The biomarker NT-pro-BNP is released from the myocardium when it is stretched, inflamed or ischemic; higher postoperative plasma concentrations after noncardiac surgery are associated with a greater 30-day risk of mortality and nonfatal myocardial infarction. ${ }^{16}$ Plasma high-sensitivity C-reactive protein is a common measure of systemic inflammation, where a higher 
postoperative concentration is associated with a greater magnitude of surgical injury. ${ }^{17}$

The secondary outcomes were acute kidney injury (based on, relative to the preoperative value, either an increase $\geq 26.5 \mu \mathrm{mol} / \mathrm{L}[\geq 0.3 \mathrm{mg} / \mathrm{dL}$ ] in the concentration of serum creatinine in the $48 \mathrm{~h}$ after surgery or an increase of $\geq 50 \%$ in the concentration of serum creatinine in the 7 days after surgery), ${ }^{18}$ hospital length of stay and a composite of 14 adjudicated clinical events within 30 days of surgery (described in section 5 of Appendix 1 and including outcomes of death, myocardial infarction and stroke). Outcomes to assess curcumin safety included diarrhea, bleeding and hypoglycemia. ${ }^{19}$

\section{Statistical analysis}

As presented in section 6 of Appendix 1, we determined that enrolling 600 patients would give the trial $80 \%$ power to detect meaningful differences in our primary biomarker outcomes. Thus, the target recruitment for this trial was 600 patients for analysis. The external data and safety monitoring committee reviewed the safety data 4 times during the trial and recommended that the trial be completed as planned. We evaluated all patients who did not withdraw their consent from trial participation according to the group to which they were assigned, consistent with a slight modification of the intention-to-treat principle (see Results section; most withdrawals occurred before the first day on which study capsules were scheduled to be taken).

We used standard statistics to describe baseline characteristics. We compared continuous variables between the 2 groups using an independent-samples $t$ test or Wilcoxon rank-sum test, and compared dichotomous variables using a $\chi^{2}$ or Fisher exact test.

To compare curcumin and placebo outcome measures, we used linear regression for continuous variables and logistic regression for binary variables. A 2-sided $p$ value of less than 0.05 indicated statistical significance, without adjustment for multiple comparisons. We decided that if curcumin was associated with significant improvement in at least 2 of our 4 primary biomarker outcomes, with no evidence of harm in the remaining outcomes, we would conclude that curcumin exerts a positive biological effect. The chance of observing statistical significance (in any direction) on at least 2 of the 4 biomarker outcomes was $1.4 \%$ if, in truth, there was no biological effect of curcumin. We imputed missing continuous variables using multiple imputations with monotone linear regression, and did the same for missing binary variables using monotone logistic regression.

We completed 3 additional analyses. We adjusted for the following relevant clinical baseline characteristics in the regression models: age; sex; body mass index; estimated glomerular filtration rate; presence of congestive heart failure, coronary artery disease or a previous cerebrovascular event; history of smoking; type of repair planned; and baseline medication use (angiotensin-converting enzyme inhibitors or angiotensin II receptor blockers, diuretics, nonsteroidal anti-inflammatory drugs and statins). We also performed a complete case analysis using the same outcome definitions and covariable adjustments as the imputed models. Finally, we explored the effect of surgery type (open v. endovascular) by including an interaction term with treatment assignment in the models.

All analyses were performed with the use of SAS software, version 9.3 (SAS Institute Inc.).

\section{Ethics approval}

Ethics approval was granted by the institutional review board at each participating site, with the initial granting body being Western University Health Sciences Research Ethics Board. An independent data safety and monitoring board oversaw the trial's safety and ethical conduct.

\section{Results}

\section{Patient enrollment and baseline characteristics}

Between November 2011 and November 2014, we randomly assigned 624 participants to receive curcumin $(n=313)$ or placebo ( $n=311$ ) (Figure 1 ; section 7 of Appendix 1 ). A total of 16 patients ( 7 in the curcumin group, 9 in the placebo group) were withdrawn before the scheduled first day of study capsule intake, and 2 patients (both in the curcumin group) had their data withdrawn for other reasons, which left 606 patients in the modified intention-to-treat population of curcumin $(n=304)$ versus placebo $(n=302)$. The last 30 -day follow-up visit after surgery occurred on Jan. 2, 2015, after which time clinical outcomes were adjudicated and biosamples were sent to a reference laboratory at Yale University, New Haven, Conn., for analysis.

A total of $10(2 \%)$ of the 606 patients died within 30 days of surgery ( 5 curcumin, 5 placebo), and all but 1 of the remaining patients completed their 30-day follow-up after surgery to determine whether any clinical events had occurred after hospital discharge. Missing data were minimal among the 606 patients; completeness of the 4 primary biomarker measures ranged from $96.5 \%$ to $99.8 \%$.

The 2 study groups were well balanced on all baseline characteristics (Table 1). The median age was 76 years, 501 (83\%) of the patients were men, 591 (98\%) were of white race, 145 (24\%) had diabetes, 194 (32\%) had an estimated glomerular filtration rate less than $60 \mathrm{~mL} / \mathrm{min}$ per $1.73 \mathrm{~m}^{2}$, and $286(47 \%)$ of the patients underwent an open procedure.

More than $85 \%$ of patients in the curcumin and placebo groups took more than $80 \%$ of their scheduled trial capsules, as determined by patient self-report combined with pill counts performed by the research coordinators (for use at each time point, see section 8 of Appendix 1 ). In the curcumin group, curcumin metabolites were detected in the urine using high-performance liquid chromatography and mass spectrometry, as described in section 9 of Appendix 1, at a concentration significantly higher than detected in the placebo group.

Other than the trial capsules, co-interventions (procedures and treatments) in the perioperative period were similar between the 2 groups (Table 2). These similarities included the 
use of prophylactic antibiotics, the volume of intravenous fluid administered, the type of anesthesia, the use of intravenous contrast agent and whether certain blood pressure medications or diuretics were used in the 24 hours before surgery.

\section{Primary outcomes}

Primary outcomes are presented in Table 3, and the preoperative and postoperative values for serum and plasma biomarkers are presented in section 10 of Appendix 1. Except for serum

\section{Enrolment}
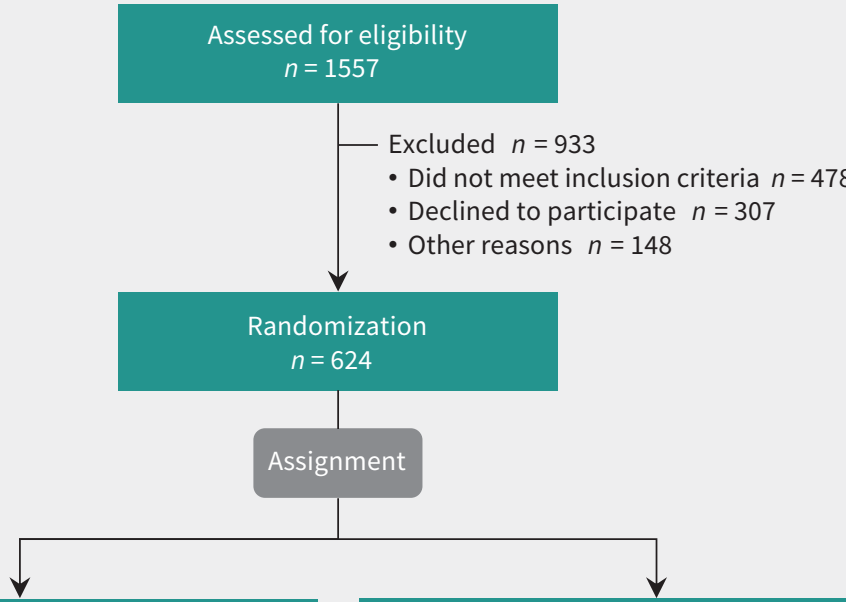

Assigned to curcumin intervention $n=313$

Assigned to placebo $n=311$

- Received assigned intervention $n=306$

- Did not receive assigned intervention

(excluded before repair, before any

scheduled capsules were to be taken) $n=7$

- Surgery date changed, postponed or cancelled and patient unable to participate in trial $n=2$

- Patient changed mind about trial participation (sometimes in setting of surgery date change, postponement or cancellation) $n=3$

- Patient's physician decided patient should not participate in trial $n=2$
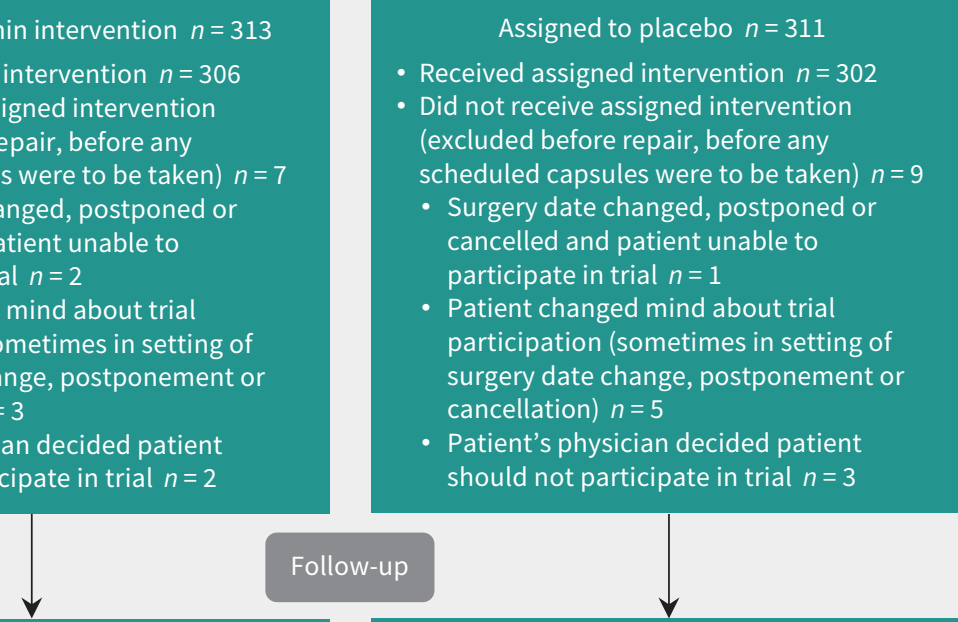

Follow-up

Lost to follow-up $n=1$

- For technical reasons the AAA was not repaired during surgery, and no data were available

Discontinued intervention $n=1$

- Participant changed mind about trial participation (for no medical reason) and asked that data be withdrawn from trial

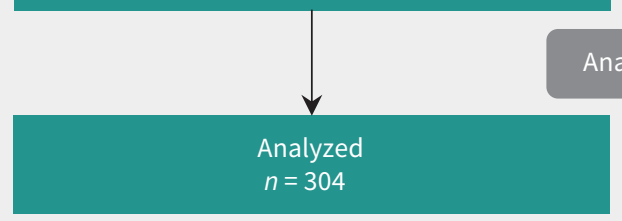

Analysis

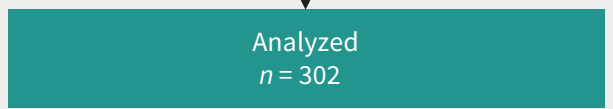

Figure 1: Patient enrolment, allocation and follow-up. Note: AAA = abdominal aortic aneurysm. 
Table 1: Characteristics of the patients at baseline

Study group; no. (\%) of patients $\dagger$

Characteristic

\section{Demographic}

Age, yr, median (IQR)

Sex, women

Ethnicity, white

\section{Comorbidities}

Abdominal aortic aneurysm, mm diameter, median (IQR)

Current smoker

Diabetes mellitus

Congestive heart failure

Hypertension

Chronic obstructive pulmonary disease

Coronary artery disease, including angina

Prior stroke or transient

ischemic attack

Gastroesophageal reflux disease

\section{Medications}

ACE-I or ARB

Diuretic

Calcium channel blocker

$\beta$-Blocker

Antiplatelet drug, including ASA

Anticoagulant

Oral hypoglycemic

Insulin

Statin

\section{Physical examination}

Weight, kg, median (IQR)

Body mass index $>35 \mathrm{~kg} / \mathrm{m}^{2}$

Systolic blood pressure,

$\mathrm{mm} \mathrm{Hg}$

Diastolic blood pressure,

$\mathrm{mm} \mathrm{Hg}$

\section{Laboratory investigations:} eGFR, $\mathrm{mL} / \mathrm{min}$ per $1.73 \mathrm{~m}^{2}$

$\begin{array}{lcc}45 \text { to }<60 & 61(20) & 55(18) \\ 30 \text { to }<45 & 34(11) & 24(8) \\ <30 & 12(4) & 8(3)\end{array}$

Note: $A C E-I$ = angiotensin-converting enzyme inhibitor, $A R B=$ angiotensin II receptor blocker, ASA = acetylsalicylic acid, eGFR = estimated glomerular filtration rate assessed with the Chronic Kidney Disease Epidemiology (CKD Epi) equation, $\mathrm{IQR}=$ interquartile range.

*Except where indicated otherwise.

†There were no significant differences between the 2 groups for any of the characteristics.

$47(16)$

$293(97)$

57 (54 to 61$) \quad 57(55$ to 60$)$

$94(31)$

$92(30)$

73 (24)

$72(24)$

14 (5)

$13(4)$

$238(78)$

$224(74)$

$86(28)$

$184(61)$

$182(60)$

$42(14)$

$35(12)$

74 (24)

73 (24)

$178(59)$

$184(61)$

$101(33)$

$90(30)$

94 (31)

$83(27)$

$118(39)$

$128(42)$

$211(69)$

$191(63)$

$18(6)$

$20(7)$

$61(20)$

$58(19)$

$6(2)$

7 (2)

$212(70)$

209 (69)

$\begin{array}{cc}83 \text { (71 to } 94) & 86 \text { (74 to } 98) \\ 25(8) & 34(11) \\ 134(122 \text { to } 146) & 134 \text { (122 to } 146)\end{array}$

79 (70 to 85$) \quad 78$ (70 to 83$)$

(3)
Table 2: Non-study drug procedures and treatments used in the perioperative period
Procedure or treatment

\section{Curcumin} $n=304$

\section{IV fluids and antibiotics}

Before repair

Volume of fluids given

During repair

Volume of fluids given, $\mathrm{L}$, median (IQR)

Colloids

$64(21)$

Mannitol

$19(6)$

Furosemide

$5(2)$

Inotrope or vasopressor

$175(58)$

Medications received in $\mathbf{2 4} \mathrm{h}$

before repair

ACE-I or ARB

84 (28)

Oral diuretic

39 (13)

Antiplatelet drug, including ASA

$127(42)$

Type of anesthesia

General

$265(87)$

Neuraxial

$166(55)$

\section{Surgical procedure performed}

Endovascular

$153(50)$

Open, or endovascular and open

$151(50)$

Use of suprarenal clamp

27 (9)

Use of IV contrast

$153(50)$

Renal artery revascularization

29 (10)

Lower limb revascularization

35 (12)

Medications received in $\mathbf{2 4} \mathrm{h}$

after repair

ACE-I or ARB

90 (30)

Oral diuretic

$\begin{array}{lcr}\text { None } & 102(34) & 100(33) \\ <100 \mathrm{~mL} & 16(5) & 18(6) \\ 100 \text { to } 500 \mathrm{~mL} & 139(46) & 138(46) \\ >500 \mathrm{~mL} & 47(15) & 46(15) \\ \text { Sodium bicarbonate } & 12(4) & 9(3) \\ \text { Prophylactic antibiotics } & 254(84) & 258(85) \\ \text { Vancomycin } & 16(6) & 18(6) \\ \text { Aminoglycoside } & 13(4) & 14(5)\end{array}$

$57(19)$

$16(5)$

$5(2)$

$190(63)$

$91(30)$

40 (13)

$120(40)$

$275(91)$

$140(46)$

$167(55)$

$135(45)$

$19(6)$

$166(55)$

$22(7)$

$31(10)$

84 (28)

Antiplatelet drug, including ASA

$205(67)$

$214(71)$

Note: $\mathrm{ACE}-\mathrm{I}$ = angiotensin-converting enzyme inhibitor, $\mathrm{ARB}=$ angiotensin II receptor blocker, ASA = acetylsalicylic acid, IQR = interquartile range, IV = intravenous.

*Except where indicated otherwise.

†There were no significant differences between the 2 groups for any of the characteristics. 
Table 3: Primary, secondary and safety outcomes

\begin{tabular}{|c|c|c|c|}
\hline \multirow[b]{2}{*}{ Outcome } & \multicolumn{2}{|c|}{ Study group; no. (\%) of patients* } & \multirow[b]{2}{*}{$p$ value } \\
\hline & $\begin{array}{c}\text { Curcumin } \\
n=304\end{array}$ & $\begin{array}{c}\text { Placebo } \\
n=302\end{array}$ & \\
\hline \multicolumn{4}{|l|}{ Primary† } \\
\hline Postoperative urine IL-18, pg/mL, median (IQR) & $13(6$ to 27$)$ & $16(7$ to 30$)$ & 0.2 \\
\hline \multicolumn{4}{|l|}{ Perioperative rise (postoperative minus preoperative) } \\
\hline Serum creatinine, $\mu \mathrm{mol} / \mathrm{L}$, median (IQR) & $1(-7$ to 19$)$ & 1 (-6 to 12$)$ & 0.2 \\
\hline Plasma NT-pro-BNP, mesoscale pg/mL, median (IQR) & 221 (67 to 511$)$ & 184 (48 to 431$)$ & 0.1 \\
\hline Plasma high-sensitivity C-reactive protein, $\mu \mathrm{g} / \mathrm{mL}$, median (IQR) & 58 (28 to 95$)$ & 58 (30 to 90$)$ & 0.9 \\
\hline \multicolumn{4}{|l|}{ Secondary } \\
\hline Acute kidney injury & $51(17)$ & $30(10)$ & 0.01 \\
\hline Hospital length of stay, $d$, median (IQR) & $5(2$ to 8$)$ & $5(2$ to 7$)$ & $>0.9$ \\
\hline Composite of clinical events & $28(9)$ & $27(9)$ & 0.9 \\
\hline \multicolumn{4}{|l|}{ Outcomes to assess curcumin safety $\ddagger$} \\
\hline Change in hemoglobin (postoperative minus preoperative), $\mathrm{g} / \mathrm{L}$, median (IQR) & $-33(-46$ to -23$)$ & $-32(-43$ to -24$)$ & 0.6 \\
\hline Clinically important bleeding & $7(2)$ & $10(3)$ & 0.4 \\
\hline Peptic ulcer & $2(1)$ & $1(<1)$ & 0.6 \\
\hline Glucose $0-3 \mathrm{~h}$ after repair, mmol/L, median (IQR) & 7 (6 to 9$)$ & 7 (6 to 9$)$ & 0.3 \\
\hline Hypoglycemic event & $9(3)$ & $3(1)$ & 0.1 \\
\hline Diarrhea & $16(5)$ & $20(7)$ & 0.5 \\
\hline Any nausea & $31(10)$ & $25(8)$ & 0.4 \\
\hline
\end{tabular}

creatinine, the postoperative biomarkers were measured at a median (IQR) of 1 ( 1 to 1 ) hour after the end of the repair. For both curcumin and placebo groups combined, there was a $202 \%$ rise in the mean perioperative concentration of plasma NT-pro-BNP ([postoperative minus preoperative value]/ preoperative value) and a $1846 \%$ rise in the mean perioperative concentration of plasma high-sensitivity C-reactive protein. Relative to placebo, curcumin did not significantly affect any of the 4 biomarker concentrations (curcumin v. placebo, median postoperative urine interleukin-18 13 v. $16 \mathrm{pg} / \mathrm{mL}, p=$ 0.2; median perioperative [postoperative minus preoperative] rise in serum creatinine, $1 \mathrm{v} .1 \mu \mathrm{mol} / \mathrm{L}, p=0.2$; median perioperative rise in plasma NT-pro-BNP, 221 v. $184 \mathrm{pg} / \mathrm{mL}, p=0.1$; and median perioperative rise in plasma high-sensitivity C-reactive protein, 58 v. $58 \mu \mathrm{g} / \mathrm{mL}, p=0.9)$.

\section{Secondary outcomes}

Secondary outcomes are also presented in Table 3. There was a higher risk of acute kidney injury with curcumin than with placebo (51/304 [17\%] v. 30/302 [10\%], $p=0.01)$, and no difference between the 2 groups in the median length of hospital stay ( 5 v. 5 days, $p>0.9$ ) or the risk of a composite of clinical events ([including death, myocardial infarction and stroke, as described in section 5 of Appendix 1], 28/304 [9\%] v. $27 / 302[9 \%], p=0.9)$.

\section{Safety outcomes}

Outcomes used to assess curcumin safety are also presented in Table 3. There were no statistically significant differences in the likelihood of these outcomes between the curcumin and placebo groups, including the risk of clinically important bleeding (7/304 [2\%] v. 10/302 [3\%], $p=0.4)$, hypoglycemia (9/304 [3\%] v. 3/302 [1\%], $p=0.1)$ or diarrhea $(16 / 304$ [5\%] v. 20/302 [7\%], $p=0.5)$.

\section{Additional analyses}

No effect of curcumin versus placebo on study outcomes was consistent in our 3 additional analyses, as described in section 11 of Appendix 1, including the adjusted analysis, analysis restricted to patients with complete data and analysis with both subgroups defined by type of repair (open or endovascular). Furthermore, there was no meaningful betweengroup difference in kidney function at 90 days, as described in section 12 of Appendix 1. 


\section{Interpretation}

In this study, perioperative oral curcumin in patients undergoing elective abdominal aortic aneurysm repair had no beneficial effect on a biomarker panel assessing injury and inflammatory response, hospital length of stay or a composite of clinical events.

Our findings are discordant from a prior single-centre trial, in which curcumin (compared with placebo) attenuated the rise in concentrations of 3 biomarkers after coronary artery bypass graft surgery. ${ }^{10}$ We measured 2 of these 3 biomarkers in our trial, and our trial enrolled 5 times the number of patients across 10 centres in the setting of a different surgical procedure. Both trials used a total daily dose of $4 \mathrm{~g}$ for the studied treatment; however, unlike the capsules in our trial, those in the prior trial were given for a period of 8 days, were ingested 4 times a day, and included demethoxycurcumin and bisdemethoxycurcumin in addition to curcumin. It is unknown whether differences in the trial capsules or their dosing schedule explains the discordant findings between the 2 trials.

We found no statistically significant between-group difference in the perioperative rise in serum creatinine concentration; however, we did find a higher risk of clinical acute kidney injury among patients who received curcumin than among those who received placebo. It is possible that the latter was simply a chance finding in the context of multiple statistical comparisons. However, this risk should be carefully assessed in future trials of curcumin, given that adverse events with natural health products often go unrecognized. ${ }^{20}$

\section{Limitations}

Our methods to conduct, analyze and report this randomized controlled trial resulted in a low risk of bias. ${ }^{21}$ In this trial, we observed no benefit of curcumin on 4 biomarkers assessing different aspects of perioperative injury and the inflammatory response, but these biomarkers have all the limitations of surrogate outcomes. For reasons of cost, we initially measured postoperative biomarkers (except serum creatinine) right after the abdominal aortic aneurysm repair; upon observing the results of these measurements, we elected to not pursue further biomarker measurements in the samples collected in the days after the repair. Although the concentration of some markers at this first postoperative time point were very different from the preoperative values, our finding of no beneficial effect of curcumin might have differed if we had measured these markers multiple times in the days after repair. Nonetheless, given the primary and secondary outcome findings of this study, it would be difficult to justify the substantial resources needed to conduct a larger trial to determine more precisely the effects of our curcumin regimen on the perioperative outcomes that matter most to patients and their health care providers.

Our curcumin regimen of $4000 \mathrm{mg}$ a day is the average dose used in many published and registered clinical trials. We hypothesized that this dose would exert a pharmacodynamic effect. However, it remains possible that a different formulation of curcumin could have achieved a higher concentration in blood and a different trial result. Curcumin is notorious for being poorly absorbed from the gastrointestinal tract; 22 however, in our study we detected metabolites of curcumin in the urine.

\section{Conclusion}

Turmeric has been used for thousands of years in Indian and Chinese medicine, and curcumin continues to gain popularity today as a natural health supplement. In this randomized trial, the largest to date, perioperative oral curcumin did not ameliorate the complications of elective abdominal aortic aneurysm repair. Our findings emphasize the importance of testing turmeric and curcumin in rigorous human clinical trials before espousing any health benefits, as is currently done in the popular media.

\section{References}

1. Newman DJ, Cragg GM. Natural products as sources of new drugs from 1981 to 2014. J Nat Prod 2016;79:629-61.

2. Gupta SC, Patchva S, Aggarwal BB. Therapeutic roles of curcumin: lessons learned from clinical trials. AAPS J 2013;15:195-218.

3. Daniells S. Turmeric leads the charge as herbal sales continue to bloom. In: Nutralngredients-USA. West Sussex (UK): William Reed Business Media Ltd.; 2016 Sept. 21 [updated 2017 June 2]. Available: www.nutraingredients-usa.com /Article/2016/09/22/Turmeric-leads-the-charge-as-herbal-sales-continue-to-bloom\# (accessed 2018 Oct. 4).

4. Hewlings SJ, Kalman DS. Curcumin: a review of its' [sic] effects on human health. Foods 2017;6:pii:E92.

5. Fadus MC, Lau C, Bikhchandani J, et al. Curcumin: an age-old anti-inflammatory and anti-neoplastic agent. J Tradit Complement Med 2016;7:339-46.

6. Sahebkar A, Cicero AFG, Simental-Mendía LE, et al. Curcumin downregulates human tumor necrosis factor- $\alpha$ levels: a systematic review and meta-analysis of randomized controlled trials. Pharmacol Res 2016;107:234-42.

7. Aydin MS, Caliskan A, Kocarslan A, et al. Intraperitoneal curcumin decreased lung, renal and heart injury in abdominal aorta ischemia/reperfusion model in rat. Int $J$ Surg 2014;12:601-5.

8. Buyuklu M, Kandemir FM, Ozkaraca M, et al. Protective effect of curcumin against contrast induced nephropathy in rat kidney: What is happening to oxidative stress, inflammation, autophagy and apoptosis? Eur Rev Med Pharmacol Sci 2014;18:461-70.

9. Trujillo J, Chirino YI, Molina-Jijón E, et al. Renoprotective effect of the antioxidant curcumin: recent findings. Redox Biol 2013;1:448-56.

10. Wongcharoen W, Jai-Aue S, Phrommintikul A, et al. Effects of curcuminoids on frequency of acute myocardial infarction after coronary artery bypass grafting. Am J Cardiol 2012;110:40-4.

11. Tayyem RF, Heath DD, Al-Delaimy WK, et al. Curcumin content of turmeric and curry powders. Nutr Cancer 2006;55:126-31.

12. Kwon Y. Estimation of curcumin intake in Korea based on the Korea National Health and Nutrition Examination Survey (2008-2012). Nutr Res Pract 2014; 8:589-94.

13. Melnikov VY, Ecder T, Fantuzzi G, et al. Impaired IL-18 processing protects caspase-1-deficient mice from ischemic acute renal failure. J Clin Invest 2001; 107:1145-52.

14. Awad AS, El-Sharif AA. Curcumin immune-mediated and anti-apoptotic mechanisms protect against renal ischemia/reperfusion and distant organ induced injuries. Int Immunopharmacol 2011;11:992-6.

15. Parikh CR, Coca SG, Thiessen-Philbrook H, et al. Postoperative biomarkers predict acute kidney injury and poor outcomes after adult cardiac surgery. J Am Soc Nephrol 2011;22:1748-57. 
16. Rodseth RN, Biccard BM, Le Manach Y, et al. The prognostic value of preoperative and post-operative B-type natriuretic peptides in patients undergoing noncardiac surgery: $\mathrm{B}$-type natriuretic peptide and $\mathrm{N}$-terminal fragment of pro-B-type natriuretic peptide: a systematic review and individual patient data meta-analysis. J Am Coll Cardiol 2014;63:170-80.

17. Watt DG, Horgan PG, McMillan DC. Routine clinical markers of the magnitude of the systemic inflammatory response after elective operation: a systematic review. Surgery 2015;157:362-80.

18. KDIGO clinical practice guideline for acute kidney injury. Kidney Int Suppl 2012;2(1):1-138. Available: www.kdigo.org/clinical_practice_guidelines/pdf/ KDIGO AKI Guideline.pdf (accessed 2018 Oct. 4).
19. Melo ISV, Santos AFD, Buenoc NB. Curcumin or combined curcuminoids are effective in lowering the fasting blood glucose concentrations of individuals with dysglycemia: systematic review and meta-analysis of randomized controlled trials. Pharmacol Res 2018;128:137-44.

20. Di Lorenzo C, Ceschi A, Kupferschmidt H, et al. Adverse effects of plant food supplements and botanical preparations: a systematic review with critical evaluation of causality. Br J Clin Pharmacol 2015;79:578-92.

21. Higgins JPT, Altman DG, Gøtzsche PC, et al. The Cochrane Collaboration's tool for assessing risk of bias in randomised trials. BMJ 2011;343:d5928.

22. Anand P, Kunnumakkara AB, Newman RA, et al. Bioavailability of curcumin problems and promises. Mol Pharm 2007;4:807-18.
Competing interests: P.J. Devereaux has received grants from Abbott Diagnostics, Boehringer Ingelheim, Covidien, Octopharma, Philips Healthcare, Roche Diagnostics amd Stryker for projects outside the work reported here. Matthew James is the principal investigator on an investigatorinitiated research grant funded by Amgen Canada outside the work reported here. No other competing interests were declared.

This article has been peer reviewed.

Affiliations: Departments of Medicine (Garg, McArthur, Moist, Schumann, ThiessenPhilbrook, Weir), of Epidemiology and Biostatistics (Garg, Dubois, Moist, Weir) and of Surgery (Dubois), Western University, London, Ont.; Departments of Health Research Methods, Evidence, and Impact (Devereaux, Walsh), of Medicine (Devereaux, Walsh) and of Surgery (lyer), McMaster University, Hamilton, Ont.; Departments of Surgery (Hill) and of Medicine (Sood, Hiremath), University of Ottawa, Ottawa, Ont.; Department of Experimental Therapeutics, Cancer Medicine and Immunology (Aggarwal), The University of Texas MD Anderson Cancer Center, Houston, Tex.; Department of Surgery (Guzman), University of Manitoba, Winnipeg, Man.; Department of Medicine (James), University of Calgary, Cal- gary, Alta.; Faculté de médecine (Ouellet), Université de Montréal, Montréal, Que.; Program of Applied Translational Research, Department of Medicine (Parikh), and Section of Nephrology (Parikh), Yale University School of Medicine, New Haven, Conn.; Department of Anesthesia (Sharan), Laurentian University, Sudbury, Ont.; Department of Medicine (Tobe, Wald), University of Toronto, Toronto, Ont.; Department of Medicine (Pannu), University of Alberta, Edmonton, Alta.

Contributors: Amit Garg was the principal investigator. All of the authors were involved with the design and implementation of the trial. Amit Garg, Eric McArthur and Heather Thiessen-Philbrook performed the statistical analysis. Amit Garg and Neesh Pannu were responsible for writing the first draft of the manuscript; all authors made critical revisions to the draft for important intellectual content All of the authors gave final approval of the version to be published and agreed to be accountable for all aspects of the work. The corresponding author had full access to all data in the study and had final responsibility for the decision to submit for publication.

Funding: This study was funded by the Canadian Institutes of Health Research (ClHR), operating grant numbers 229978 and
191259. The funders of the study had no role in the study design, data collection, data analysis, data interpretation or writing of the report. Amit Garg was supported by the Dr. Adam Linton Chair in Kidney Health Analytics and a Clinician Investigator Award from the CIHR.

Data sharing: Researchers interested in accessing the trial database may contact the corresponding author.

Acknowledgements: The authors thank the members of the external data and safety monitoring committee, Drs. Duminda Wijeysundera and Vanita Jassal, for their oversight of this trial. They also thank Darek Gozdzik for his help managing the trial database, the Kidney O'Brien Center Core at Yale University (NIH P30DK79337) for measurement of the curcumin metabolites and biomarkers, and all of the research coordinators who helped collect the data. The authors thank Jade Dirk for administrative assistance in submitting the manuscript for publication, and the patients who participated in this trial.

Accepted: Sept. 6, 2018

Correspondence to: Amit Garg, amit.garg@ Ihsc.on.ca 